\title{
Análise técnica-econômica da derrubada de Eucalyptus sp. com feller-buncher: uma abordagem estocástica
}

\author{
Technical-economic analysis of the felling of Eucalyptus species with \\ feller-buncher: a stochastic approach \\ Gilberto Pereira ${ }^{1}$ (D), Paulo Torres Fenner ${ }^{1}$ (1) Gislaine Cristina Batistela² (1), Danilo Simões² (1) \\ ${ }^{1}$ Universidade Estadual Paulista, Faculdade de Ciências Agronômicas - UNESP, Botucatu, SP, Brasil \\ ${ }^{2}$ Universidade Estadual Paulista, Câmpus Experimental de Itapeva - UNESP, Itapeva, SP, Brasil
}

Como citar: Pereira, G., Fenner, P. T., Batistela, G. C., Simões, D. (2020). Análise técnica-econômica da derrubada de Eucalyptus sp. com feller-buncher: uma abordagem estocástica. Scientia Forestalis, 48(126), e3229. https://doi.org/10.18671/scifor.v48n126.14

\begin{abstract}
Resumo
Em operações de colheita de madeira mecanizada, a execução da simulação pelo método de Monte Carlo permite a obtenção de probabilidades de ocorrência dos parâmetros técnico-econômicos, consentindo descrever as incertezas e, conseguinte, a otimização dos sistemas de colheita. Diante disso, objetivou-se a abordagem estocástica da derrubada de florestas plantadas com Eucalyptus sp. realizada por uma máquina autopropelida com cabeçote de corte com disco feller-buncher, em dois espaçamentos entre árvores, por meio do método de simulação de Monte Carlo para a análise dos parâmetros técnicoeconômicos. Foram ponderados dados históricos sobre valores monetários ocorridos, volume de madeira derrubada e o tempo efetivo de produção, consideradas variáveis sob condições de incertezas, as quais foram ajustadas por meio de distribuições de probabilidades. O método de simulação Monte Carlo foi aplicado aos modelos matemáticos para incorporar soluções estocásticas. Os resultados permitiram concluir que os valores monetários demandados para a substituição de peças da máquina autopropelida possui o maior grau de associação com o custo por hora programada; há uma relação não linear forte negativa entre os valores monetários necessários para a aquisição de óleos lubrificantes e graxas e entre o custo por hora programada da máquina autopropelida, evidenciando que quanto maior o investimento em lubrificação menor será o custo por hora programada desta máquina; o valor médio do custo de produção para a derrubada de Eucalyptus sp. é explicado por $46 \%$ em termos de variância da produtividade por hora efetivada máquina autopropelida.
\end{abstract}

Palavras-chave: Colheita de madeira; Avaliação de custos; Incerteza; Monte Carlo; Produtividade.

\begin{abstract}
In mechanized harvesting operations for wood, the Monte Carlo method allows the probability of occurrence of the technical-economical parameters, with descriptions of the uncertainties and, consequently, the optimization of harvesting systems. This study used the stochastic approach applied for felling forests planted with Eucalyptus species performed by a self-propelled machine having a felling head, with a feller-buncher disk, in two spacings between trees, through the Monte Carlo simulation method for the analysis of the technical-economic parameters. Historical data on monetary values were considered, as well as the volume of felled timber and actual production time, considering variables under uncertainty conditions, which were adjusted by probability distributions. The Monte Carlo simulation method was applied to mathematical models in order to incorporate stochastic solutions. The results reached the conclusion that the monetary values demanded for spare part replacements for the self-propelled machine presented the highest degree of association with the programmed hourly
\end{abstract}

Fonte de financiamento: None.

Conflito de interesse: Nada a declarar.

Autor correspondente: gilbertopereira960@gmail.com

Recebido: 19 dezembro 2018.

Aceito: 19 julho 2019.

Editor: Francides Gomes Silva Júnior

(c) (i) Este é um artigo publicado em acesso aberto (Open Access) sob a licença Creative Commons Attribution, que permite uso, distribuição e c) reprodução em qualquer meio, sem restrições desde que o trabalho original seja corretamente citado. 
cost; there was a strong negative nonlinear relationship between the monetary values required for the acquisition of lubricating oils and greases and the programmed hourly cost of the self-propelled machine, showing that the higher the investment in lubrication, the lower the programmed hourly cost of this machine; the average value of the production cost for felling Eucalyptus species is explained by $46 \%$ of the productivity variance per hour of the self-propelled machine.

Keywords: Timber harvesting; Cost assessment; Uncertainty; Monte Carlo; Productivity.

\section{INTRODUÇÃO}

As máquinas autopropelidas que possuem na parte frontal o cabeçote de corte com disco feller-buncher, são empregadas em sistemas que realizam a colheita de árvores inteiras, ou melhor, exercem o corte de derrubada das árvores, realizado próximo ao nível do solo, que, por conseguinte, são concentradas em uma mesa acumuladora para posteriormente serem dispostas em feixes de árvores no interior dos talhões, em posições intrínsecas à operação subsequente, isto é, a extração dos feixes de árvores.

Nesta acepção, o sistema de colheita de madeira com o uso de máquinas que possuem estas características técnicas, segundo Moreira et al. (2004), Silva et al. (2007), Fernandes et al. (2010) e Sant'anna (2014) foi relatada no fim da década de 70 e, vem sendo uma das mais utilizadas para a colheita de madeira, assim como, avaliadas sob diversos aspectos, dentre estes, os custos envolvidos, que de acordo com Miyajima et al. (2017), de forma abrangente, podem ser definidos como a quantificação econômica dos fatores produtivos. Todavia, uma condição presente no conhecimento dos custos de colheita de madeira é a incerteza, devido ao conjunto de fatores envolvidos ou da insuficiência de dados.

Uma das alternativas para analisar estas incertezas é a simulação pelo Método de Monte Carlo, o qual consente quantificar os riscos envolvidos por meio de distribuições de probabilidades das variáveis de interesse, como exemplo, a partir de dados históricos da operação florestal objeto de estudo. De acordo com Porras (2018) este método caracteriza-se por construir um modelo matemático com base nas amostras de um problema, obtendo desta forma uma resposta probabilística.

Portanto, a simulação pelo método de Monte Carlo envolve a amostragem aleatória de distribuições de probabilidade dentro de um modelo matemático, sendo um dos métodos numéricos mais versáteis e práticos de aproximação, que tem como propósito a análise das incertezas do mundo real por meio do impacto da aleatoriedade das variáveis dependentes (Parra et al., 2015; Kabungo \& Jenkins, 2016; Buhara \& Pehlivan, 2019).

Neste contexto, ao considerar os diversos fatores que podem influenciar o custo de produção da derrubada de árvores de Eucalyptus sp. e a possibilidade de o gestor florestal atribuir distribuições de probabilidades aos parâmetros envolvidos sob condições de incerteza, objetivou-se a abordagem estocástica da derrubada de florestas plantadas com Eucalyptus sp. realizada por uma máquina autopropelida com cabeçote de corte com disco feller-buncher, em dois espaçamentos entre árvores, por meio do método de simulação de Monte Carlo para a análise dos parâmetros técnico-econômicos.

\section{MATERIAL E MÉTODOS}

\section{Área experimental}

O estudo foi realizado a partir da série de dados técnico-econômicos de uma empresa de base florestal, do período compreendido entre julho de 2011 e agosto de 2016, intrínsecos à derrubada de florestas plantadas com Eucalyptus sp. em corte raso, implantadas a partir de mudas clonais. Destarte, a área total colhida foi de 1.096,20 hectares, que resultou em 466.954,74 metros cúbicos de madeira derrubada.

A idade média das florestas era de 6,65 $\pm 1,45$ anos, com árvores que possuíam altura média de $25,73 \pm 3,55 \mathrm{~m}$, incremento médio anual de $54,55 \mathrm{~m}^{3} \mathrm{ha}^{-1} \mathrm{ano}^{-1}$ e com produtividade média das florestas de $349,32 \mathrm{~m}^{3}$ com casca por hectare. Estas florestas estavam localizadas 
na região Centro-Oeste do Estado de São Paulo. Posto isto, nesta região, segundo Santos et al. (2018a) as unidades de solos são predominantemente do tipo Latossolo Vermelho Amarelo, Distrófico Psamítico e apresentam classe de relevo plano, com declividade que variava entre 0 e 3\% conforme o Instituto Geográfico e Cartográfico do Estado de São Paulo (2018).

\section{Operação de derrubada de Eucalyptus sp.}

A operação de derrubada foi realizada por uma única máquina autopropelida com rodados de esteiras da marca John Deere, modelo 903 k, equipada com motor de 6 cilindros com 300 HP (223 kW)a 1.900 rotações por minuto. Na parte frontal desta máquina havia um cabeçote de corte com disco feller-buncher modelo FR22B, para capacidade máxima de corte de árvores com até $559 \mathrm{~mm}$ e capacidade de acúmulo de $0,48 \mathrm{~m}^{2}$,outrossim, com alcance máximo do braço de $6,71 \mathrm{~m}$. Isto posto, o equipamento florestal foi adquirido por US\$ $496.118 \mathrm{em}$ agosto de 2010, era utilizado para a atividade fim em três turnos de oito horas e estava com 22.901 horas de uso acumuladas. Ademais, o valor modal do salário por hora dos operadores durante o período analisado, incluso benefícios e encargos sociais, foi de US\$15,32.

O eito de operação da máquina autopropelida foi composto por quatro linhas de plantio, com o deslocamento sobre a terceira linha e com os rodados nas entrelinhas. O direcionamento dos feixes foi com vistas a permitir o aumento da produtividade por hora efetiva da operação seguinte após a derrubada, ou seja, da operação de extração dos feixes das árvores de Eucalyptus sp. até as margens das estradas florestais.

Mais precisamente, a derrubada foi realizada em duas áreas florestais com condições operacionais distintas, caracterizadas em consonância ao volume médio individual (VMI)e a densidade populacional, descritas como: Área 1: espaçamento de três metros entre linhas de plantio e dois metros entre árvores, com número médio de 1.487 árvores, em suma, $89 \%$ de sobrevivência e, VMI de 0,22 m³; Área 2: espaçamento de três metros entre linhas de plantio e dois metros e meio entre árvores, com sobrevivência média de 1.318 árvores, logo, 99\% de sobrevivência e, VMI de $0,28 \mathrm{~m}^{3}$.

\section{Análise técnica}

A produtividade por hora efetivada máquina autopropelida foi determinada pela razão entre o volume de madeira derrubada e a quantidade de horas efetivas de operação (Equação 1).O volume de madeira foi obtido por meio da quantidade de árvores de Eucalyptus sp. derrubadas, mensuradas a partir do sistema de registro diário de operação do feller-buncher, o qual possuía um contador digital de pulsos, da marca Autonics, modelo LCD LA8N-BN. Isto posto, esta quantidade foi multiplicada pelo VMI, obtido por meio do inventário pré-corte.

Em relação à quantidade de horas efetivas da máquina autopropelida (Equação 1), foram ponderados os tempos despendidos com a operação fim, ou seja, os tempos demandados para a derrubada e disposição dos feixes das árvores, aferidos diariamente por meio do sistema de controle da empresa de base florestal. À vista disso, a quantidade de horas operacionais paradas decorrentes de acidentes, atrasos com transporte de pessoal, instruções de segurança e treinamento, troca de turno de trabalho, condições climáticas adversas, manutenções preventivas, manutenções corretivas, abastecimento de combustível, deslocamento entre talhões e necessidades fisiológicas, não foram consideradas para o cômputo das horas efetivamente trabalhadas.

$$
P H M=\frac{V_{\text {mad }}}{h}
$$

\footnotetext{
em que:

PHM é a produtividade por hora efetiva da máquina autopropelida $\left(\mathrm{m}^{3} \mathrm{~h}^{-1}\right)$;

$V_{\text {mad }}$ é o volume de madeira derrubada $\left(\mathrm{m}^{3}\right)$;

$h$ é a quantidade de horas efetivas da operação (h).
} 


\section{Análise econômica}

Os valores monetários foram expressos em dólar comercial americano (US\$), oficial do Banco Central do Brasil a preço de venda (Banco Central do Brasil, 2018). Logo, considerou-se como taxa de câmbio o preço da moeda estrangeira medido em unidades e frações da moeda nacional, que era de $\mathrm{R} \$ 3,3237$ em 31/03/2018.

O custo por hora programada da máquina autopropelida foi determinado a partir do gerenciamento dos custos da empresa florestal que era pautado no sistema de custeio Activity Based Costing $(\mathrm{ABC})$, tendo como diretriz o cost drivers para a gestão estratégica dos custos da operação de derrubada das florestas plantadas com Eucalyptus sp. Sendo assim, considerou-se o custo real histórico, deflacionado por meio do Índice Geral de Preços - Disponibilidade Interna (IGP-DI). Em síntese, o CHP foi o somatório dos elementos de custo (EC) estruturados da seguinte forma:

Custo da mão de obra dos operadores ( $\left.\mathrm{M}_{\mathrm{ope}}\right)$ : despesas com os operadores da máquina autopropelida, ou seja, foram inclusos os valores monetários com equipamentos de proteção individual, refeições, transporte de pessoal, plano de saúde, benefícios e encargos sociais;

Custo da mão de obra dos supervisores $\left(M_{\text {sup }}\right)$ : despesas com os supervisores e encarregados florestais, ponderando os valores monetários com equipamentos de proteção individual, refeições, transporte de pessoal, plano de saúde, benefícios e encargos sociais;

Depreciação $\left(C_{\text {dep }}\right)$ : despesa demandada para recuperar o capital aplicado para aquisição da máquina autopropelida, considerando cotas lineares;

Custo com lubrificantes $\left(C_{\text {lub }}\right)$ : foram compostos pelos gastos com óleo de motor, óleo hidráulico e graxas utilizados na máquina autopropelida;

Custo com oficina móvel $\left(C_{\text {ofi }}\right)$ : foram formados pelos custos diretos e indiretos despendidos com caminhão oficina, utilizado para a realização de reparos e manutenções a campo;

Custo com combustível $\left(\mathrm{C}_{\mathrm{com}}\right)$ : gastocom óleo diesel consumido pela máquina autopropelida;

Custo de reposição de peças $\left(C_{\text {pec }}\right)$ : gastos com filtros, peças, recuperação de componentes hidráulicos utilizados nas manutenções preventivas e corretivas da máquina autopropelida;

Custo de reparos e manutenções ( $\left.C_{\text {rem }}\right)$ : foram formados pelos gastos com prestadores de serviços necessários para assegurar o funcionamento da máquina autopropelida.

O custo de produção da máquina autopropelida (CPM) durante a derrubada de florestas plantadas com Eucalyptus sp., foi calculado conforme a Equação 2, que demonstra a razão entre o custo por hora programada e a produtividade por hora efetiva da máquina autopropelida.

$$
C P M=\frac{C H P}{P H M}
$$

em que:

$C P M$ é o custo de produção da máquina autopropelida (US\$ $\left.\mathrm{m}^{-3}\right)$;

CHP é o custo por hora programada da máquina autopropelida (US\$ $\left.\mathrm{h}^{-1}\right)$.

\section{Relação linear entre os elementos decusto e o custo por hora programada da máquina autopropelida}

Para analisar o grau de interdependência linear das variáveis quantitativas discretas, em outras palavras, a associação linear entre os EC e o CHP, empregou-se o coeficiente de correlação linear de Pearson, denotado por $r$, e de acordo com Triola (2015) o valor de $r$ está sempre entre -1 e 1 . Diante disso, o coeficiente de correlação linear foi interpretado em 
consonância à Sullivan (2017), descrito como: relação linear perfeita positiva $(r=1)$, relação linear forte positiva $(0,9 \leq r<1)$, relação linear moderada positiva $(0,4 \leq r<0,9)$, relação linear forte negativa $(r=-1)$, relação linear forte negativa $(-0,9 \leq r<-1)$, relação linear moderada negativa $(-0,4 \leq r<-0,9)$.

\section{Incorporação do risco técnico-econômico}

O método de simulação de Monte Carlo foi aplicado aos modelos matemáticos, em outros termos, modelos estocásticos com distribuições de probabilidades atribuídas aos parâmetros sob condições de incerteza e com correlações entre as variáveis, para a obtenção de um conjunto de valores amostrais obtidos a partir de 100.000 iterações, realizadas por meio do software @Risk 7.6 Copyright @ 2018 (Palisade Corporation, 2018). O gerador de números aleatórios empregado foi o Mersenne Twister, outrossim, assegurou-se o mesmo parâmetro inicial para os modelos matemáticos executados.

Os parâmetros de entrada dos modelos matemáticos(inputs), consideradas como variáveis estocásticas após as atribuições de probabilidades, foram: $M_{\text {ope, }}, M_{\text {sup }}$, $C_{\text {dep }}, C_{\text {lub }}, C_{\text {ofir }}, C_{\text {com }}, C_{\text {pec }}, C_{\text {rem }}$, quantidade de horas efetivas de operação e o volume de madeira derrubada. A partir da série dos valores históricos destes inputs, foi adotado o teste de Kolmogorov-Smirnov (KS), ao nível de $1 \%$ de significância para verificar a normalidade dos dados. Posteriormente, foram ajustadas distribuições de probabilidade pelo critério de seleção Bayes Information Criterium (BIC) e, por conseguinte, foram construídos os modelos estocásticos para expressar o CHP, a PHM e o CPM de derrubada de Eucalyptus sp. com máquina autopropelida e cabeçote de corte com disco feller-buncher, caracterizados como outputs.

E por fim, com vistas a mensurar o coeficiente de correlação entre os inputs e outputs, adotou-se o coeficiente de correlação de postos de Spearman $\left(\rho_{s}\right)$ devido a estas variáveis possuírem relações não lineares e, sobretudo, de acordo Rachev et al. (2010) em finanças, particularmente em análise de riscos, é importante ter uma medida de dependência que se concentre apenas no comportamento de duas variáveis. Diante deste propósito, ponderou-se o nível de significância de 5\%.

\section{RESULTADOS E DISCUSSÃO}

\section{Análise da relação linear dos elementos de custo}

Ao considerar a derrubada de Eucalyptus sp.na Área 1 (Tabela 1), constatou-se correlação linear moderada positiva entre o $C_{\text {ofi }}$ e $C_{\text {dep }}(r=0,89)$, seguido do $C_{\text {pec }}$ e o $C_{\text {com }}(r=0,88)$ e entre a $C_{\text {dep }} \mathrm{e}$ o $\mathrm{M}_{\text {ope }}(r=0,80)$. Apesar destas correlações não apresentarem significância estatística, estas variáveis podem influenciar positivamente o CHP. Neste âmbito, devem ser adotadas ações mitigadoras para redução dos valores monetários do CHP.

Não obstante, há uma correlação negativa forte entre o $C_{\text {rem }}$ o $C_{\text {lub }}(r=-0,98)$, contudo, não é estaticamente significativa. Além disso, também há uma correlação moderada negativa $(r=-0,82)$ entre $\circ C_{\text {lub }}$ e $\circ C_{\text {ofi, }}$ as quais apresentaram significância estatística $(p<0,05)$, corroborando que a correta lubrificação e a manutenção preditiva dos componentes mecânicos e hidráulicos das máquinas autopropelidas podem diminuir os dispêndios com a oficina móvel utilizada como apoio às operações de colheita de madeira, pois de acordo com Daily \& Peterson (2017) as manutenções preditivas minimizam os requisitos de estoque de peças, além da expressiva redução de custos de manutenção. 
Tabela 1. Matriz do coeficiente de correção de Pearson entre os elementos de custo da máquina autopropelida com cabeçote de corte com disco feller-buncher durante a derrubada de Eucalyptus sp. na Área 1.

\begin{tabular}{|c|c|c|c|c|c|c|c|c|}
\hline EC & Mope & Msup & Cdep & Club & Cofi & Ccom & Cpec & Crem \\
\hline Mope & 1 & & & & & & & \\
\hline$M_{\text {sup }}$ & $0,68^{n s}$ & 1 & & & & & & \\
\hline$C_{\text {dep }}$ & $0,80^{\mathrm{ns}}$ & $0,36^{\mathrm{ns}}$ & 1 & & & & & \\
\hline$C_{\text {lub }}$ & $-0,43$ ns & $-0,09 \mathrm{~ns}$ & $-0,72$ ns & 1 & & & & \\
\hline$C_{\text {ofi }}$ & $0,77^{\text {ns }}$ & 0,22 ns & 0,89 * & $-0,82$ * & 1 & & & \\
\hline $\mathrm{C}_{\mathrm{com}}$ & $0,42^{\text {ns }}$ & $0,66^{\mathrm{ns}}$ & $0,39 \mathrm{~ns}$ & $-0,30 \mathrm{~ns}$ & $0,33^{\mathrm{ns}}$ & 1 & & \\
\hline$C_{\text {pec }}$ & $-0,27$ ns & $0,44^{n s}$ & $-0,31 \mathrm{~ns}$ & $0,00 \mathrm{~ns}$ & $-0,41 \mathrm{~ns}$ & 0,39 ns & 1 & \\
\hline $\mathrm{C}_{\mathrm{rem}}$ & $0,25^{\mathrm{ns}}$ & $0,53^{\mathrm{ns}}$ & $0,47^{\mathrm{ns}}$ & $-0,98^{n s}$ & $0,62^{\mathrm{ns}}$ & $0,88^{n s}$ & 0,91 ns & 1 \\
\hline
\end{tabular}

* - significativo a 5\%; ns - não significativo.

Em análise a derrubada de Eucalyptus sp. na Área 2 (Tabela 2), constatou-se que o maior grau de correlação linear positiva foi entre $\circ C_{\text {rem }}$ o $C_{\text {pec }}$ o qual é considerado moderado $(r=0,78)$, com significância estatística $(p<0,05)$. Verificou-se também que houve uma correlação moderada entre $\circ C_{\text {ofi }}$ e $\circ C_{\text {dep }}(r=0,75)$ e entre $\circ C_{\text {dep }} \mathrm{e} \circ \mathrm{M}_{\text {sup }}(r=0,65)$, estatisticamente significativas $(p<0,01)$. $O C_{\text {pec }}$ e o $C_{\text {com }}$ apresentaram correlação positiva de 0,52 considerada fraca com $p<0,05$.Sob o mesmo ponto de vista, os demais EC da máquina autopropelida em operação na Área 2, apresentaram valores de correlação linear inferiores a0,50, consideradas como correlações fracas ou até mesmo ínfimas, demais, não foram estatisticamente significativos.

Tabela 2. Matriz do coeficiente de correção de Pearson entre os elementos de custo da máquina autopropelida com cabeçote de corte com disco feller-buncher durante a derrubada de Eucalyptus sp. na Área 2.

\begin{tabular}{ccccccccc}
\hline EC & Mope & Msup & Cdep & Club & Cofi & Ccom & Cpec & Crem \\
\hline$M_{\text {ope }}$ & 1 & & & & & & & \\
$M_{\text {sup }}$ & $0,47^{\text {ns }}$ & 1 & & & & & & \\
$C_{\text {dep }}$ & $0,34^{\text {ns }}$ & $0,65^{* *}$ & 1 & & & & & \\
$C_{\text {lub }}$ & $0,02^{\text {ns }}$ & $-0,15^{\text {ns }}$ & $-0,27^{\text {ns }}$ & 1 & & & & \\
$C_{\text {ofi }}$ & $0,38^{\text {ns }}$ & $0,35^{\text {ns }}$ & $0,75^{* *}$ & $-0,21^{\text {ns }}$ & 1 & & & \\
$C_{\text {com }}$ & $0,16^{\text {ns }}$ & $-0,04^{\text {ns }}$ & $-0,42^{\text {ns }}$ & $0,50^{*}$ & $-0,24^{\text {ns }}$ & 1 & & \\
$C_{\text {pec }}$ & $0,30^{\text {ns }}$ & $-0,06^{\text {ns }}$ & $-0,11^{\text {ns }}$ & $0,35^{\text {ns }}$ & $-0,02^{\text {ns }}$ & $0,52^{*}$ & 1 & \\
$C_{\text {rem }}$ & $0,21^{\text {ns }}$ & $0,02^{\text {ns }}$ & $-0,45^{\text {ns }}$ & $0,16^{\text {ns }}$ & $0,31^{\text {ns }}$ & $0,55^{\text {ns }}$ & $0,78^{* *}$ & 1 \\
\hline
\end{tabular}

** e * - significativos a 1 e $5 \%$, respectivamente; ns - não significativo

\section{Ajuste das distribuições de probabilidades dos elementos de custo}

Quanto aos ajustes das distribuições de probabilidades dos EC da derrubada de Eucalyptus sp. na Área 1, 75\% dos EC, representados por $C_{\text {lub, }} C_{\text {ofi }} C_{\text {pec, }}, M_{\text {ope, }} M_{\text {sup }}$ obtiveram os melhores ajustes por meio do modelo de distribuição Triangular, caracterizada por valores mínimo, máximo e mais provável para cada EC. De acordo com Nguyen \& McLachlan (2016a) e Nguyen \& McLachlan (2016b) a distribuição Triangular pertence a uma classe de probabilidades bem conhecida e frequentemente utilizadas, além disso, de acordo Glickman \& Xu (2008) é uma maneira de envolver os gerentes ou especialistas no processo analítico, considerando suas estimativas subjetivas dos valores mínimos, mais prováveis e máximos das variáveis aleatórias.

Para os demais EC desta condição de operação $\left(C_{\text {com }}\right.$ e $\left.C_{\text {dep }}\right)$, as distribuições ajustadas foram a PERT, que também confere aos gestores florestais a atribuição dos valores mínimo, máximo e provável aos EC. Conforme Kamiya et al. (2016) a distribuição PERT é comumente usada para aproximar a distribuição em torno do valor médio com valores mínimos e 
máximos obtidos a partir da opinião de especialistas. No entendimento de Ghorabaee et al. (2017) esta é uma distribuição flexível para modelar as opiniões de especialistas e, pode ser vista como uma versão suave da distribuição uniforme ou da distribuição Triangular. Sob esta perspectiva, se não houverem séries de dados que permitam os ajustes das distribuições, pode ser aplicada de forma empírica esta distribuição aos EC, pautando-se em valores que poderão ser expressos por meio da opinião dos especialistas, a exemplo, pelos gestores florestais.

Ao interpretar os EC da máquina autopropelida durante a derrubada de Eucalyptus sp.na Área 2, dos oito EC, somente o $\mathrm{M}_{\text {sup }}$ teve o melhor ajuste por meio da distribuição PERT. De acordo com Jing et al. (2013) os resultados gerados a partir desta distribuição, se assemelham com uma probabilidade realista. Em relação aos demais EC, portanto, $C_{\text {com, }} C_{\text {dep }}$ $C_{\text {lub }}, C_{\text {offi, }} C_{\text {pec, }} C_{\text {rem e }} M_{\text {ope, }}$ que representaram $87,5 \%$ dos EC, tiveram os melhores ajustes de distribuições pelo modelo Triangular.

\section{Análise estocástica da produtividade por hora efetiva da máquina autopropelida}

Ao decompor a PHM, foi possível demonstrar que para a derrubada de Eucalyptus sp. na Área 1 o pressuposto de normalidade foi rejeitado $(K S=0,0296)$. A distribuição de probabilidade ajustada foi a Beta(BIC=886.910,52), com assimetria fraca e negativa e, com cauda mais longa à esquerda $(A=0,10)$ e grau de curtose platicúrtica $(K=4,13)$ em torno do valor médio, indicando segundo Miguel et al. (2014) a tendência de maior concentração dos dados em relação ao ponto central (Figura 1).

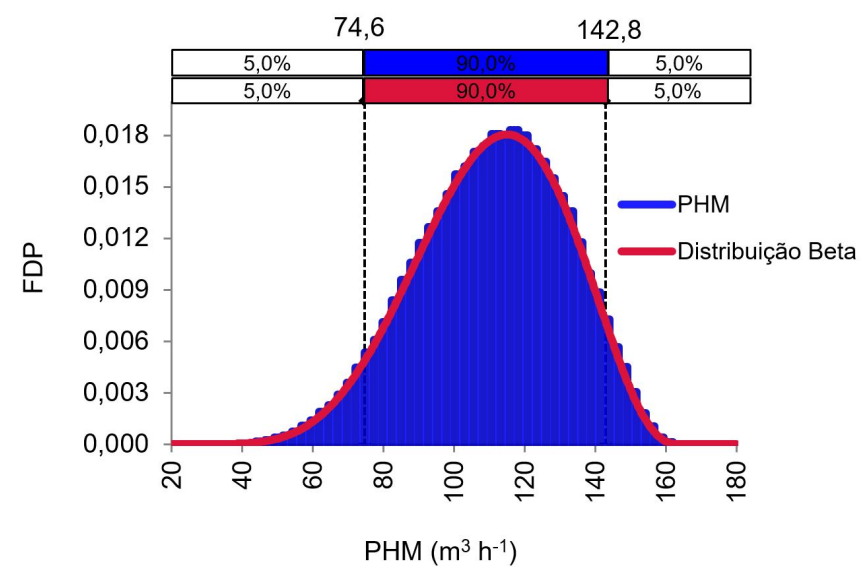

Figura 1. Função densidade de probabilidade (FDP) da produtividade por hora efetiva da máquina autopropelida com cabeçote de corte com disco feller-buncher durante a derrubada de Eucalyptus sp. na Área 1.

Assim, o valor médio da PHM foi $109,78 \mathrm{~m}^{3} \mathrm{~h}^{-1}$ e desvio padrão de $20,96 \mathrm{~m}^{3} \mathrm{~h}^{-1}$, sendo este superior aos resultados obtidos por Simões et al. (2010) os quais analisaram a PHM de uma máquina autopropelida com cabeçote de corte com disco feller-buncher em diferentes condições de operacionais, contudo, em florestas plantadas com Eucalyptus sp. em espaçamentos de $3 \mathrm{~m} \times 2 \mathrm{~m}$ e obtiveram produtividades operacionais que variaram entre $72,52 \mathrm{~m}^{3} \mathrm{PHM}^{-1} \mathrm{e} 91,60 \mathrm{~m}^{3} \mathrm{~h}^{-1}$.

Em observação à PHM durante a derrubada de Eucalyptus sp. na Área 2 rejeitou-se a hipótese de que os valores seguem uma distribuição Normal (KS=0,0190), a qual tem uma curva simétrica em torno da média, conseguinte, apresentou assimetria fraca e positiva com cauda mais longa à direita $(A=0,47)$ conforme a Figura 2 e grau de curtose platicúrtica $(K=3,38)$. Logo, o melhor ajuste para os valores observados foi a distribuição de probabilidade Gamma(BIC $=1.017 .362,50)$ com valor médio de $123,52 \mathrm{~m}^{3} \mathrm{~h}^{-1} \mathrm{e}$ desvio padrão de $39,80 \mathrm{~m}^{3} \mathrm{~h}^{-1}$. 


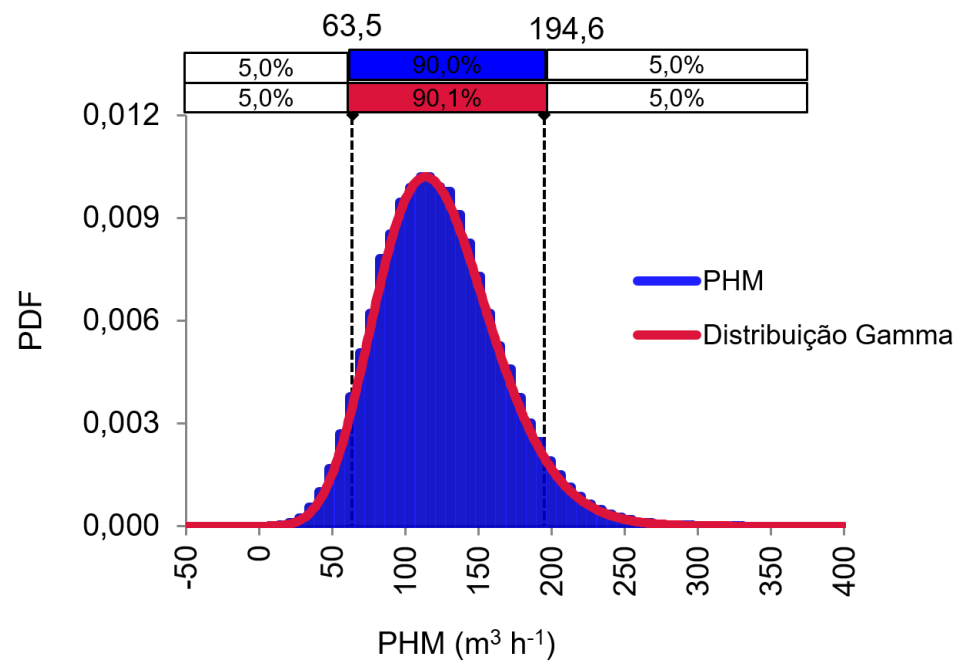

Figura 2. Função densidade de probabilidade (FDP) da produtividade por hora efetiva da máquina autopropelida com cabeçote de corte com disco feller-buncher durante a derrubada de Eucalyptus sp. na Área 2.

Todavia, Mauya et al. (2011), Alam et al. (2013), Miyajima et al. (2016), Acosta et al. (2017) e Bilici et al. (2018) ao avaliarem a colheita de madeira com máquinas autopropelidas com cabeçote de corte com disco feller-buncher, obtiveram em seus estudos valores de PHM que variaram entre 80 e $158 \mathrm{~m}^{3} \mathrm{~h}^{-1}$. Contudo, a variabilidade entre os valores da PHM pode ser explicada por diversas razões, como características técnicas da máquina autopropelida e cabeçote de corte com disco feller-buncher, ângulo de disposição dos feixes, tempo de experiência profissional dos operadores da máquina autopropelida, condições do terreno, espécie, volume individual das árvores, índice de mortalidade de plantio florestal, dentre outros, que justificam o monitoramento constante da produtividade por hora efetiva, com vistas à otimização da operação.

Ao comparar a diferença do valor médio da PHM $\left(13,74 \mathrm{~m}^{3} \mathrm{~h}^{-1}\right)$ de derrubada de Eucalyptus sp.na Área 1 e na Área 2, esta pode ser explicada devido ao percentual de sobrevivência das árvores entre as áreas experimentais, que foi de $10 \%$ e, sobretudo, devido ao VMI, que Área 2 o VMI era de $0,28 \mathrm{~m}^{3}$, o qual foi maior quando comparado a Área 1, que obteve um VMI de $0,22 \mathrm{~m}^{3}$. A influência do VMI sobre a produtividade por hora efetiva da máquina autopropelida com cabeçote de corte feller-buncher também foi confirmada por estudos realizados por Fernandes et al. (2009) e Fiedler et al. (2008).

A correlação de postos de Spearman é uma técnica não paramétrica, que permite avaliar a associação não linear entre duas variáveis a partir de dados de uma amostra aleatória. Deste modo, ao testar a associação entre o $\mathrm{V}_{\text {mad }}$ a PHM em florestas plantadas florestas com Eucalyptus sp.na Área 1, constatou-se que existe uma correlação não linear forte positiva $\left(\rho_{s}=0,94\right)$ e, uma correlação não linear fraca negativa $\left(\rho_{s}=-0,31\right)$ entre o tempo dispendido para a operação e a PHM.

Para a derrubada de Eucalyptus sp. na Área 2, verificou-se a existência de uma correlação não linear moderada positiva $\left(\rho_{s}=0,78\right)$ entre $\circ V_{\operatorname{mad}}$ e a PHM e, uma correlação não linear moderada negativa $\left(\rho_{s}=-0,56\right)$ entre o tempo efetivo dispendido e a PHM. Estas correlações não lineares, corroboram para ambas as áreas experimentais, que a quantidade de horas trabalhada da máquina autopropelida é a variável estocástica que mais influenciou a produtividade por hora da máquina autopropelida.

\section{Análise estocástica do custo por hora programada da máquina autopropelida}

Para a análise estocástica do CHP nas duas áreas experimentais estudadas, foram utilizados os valores dos coeficientes de correlação linear de Pearson entre os EC, descritos anteriormente por meio das matrizes de correlações (Tabela 1 e 2). Portanto, ao executar a 
simulação pelo Monte Carlo foram adicionados os coeficientes de correlação entre os EC devido às suas dependências, dado que os valores da distribuição de um input afetam os valores da distribuição de outro input do CHP.

Neste sentido, ao analisar o CHP da derrubada de Eucalyptus sp. na Área 1, constatou-se que os valores não seguem a uma distribuição Normal $(K S=0,0284)$. Ademais, a distribuiç̧ão de probabilidade que melhor se ajustou foi a $\operatorname{Beta}(\mathrm{BIC}=955.775,21)$ a qual resultou em assimetria fraca e positiva $(A=0,47)$, com cauda mais longa à direita e, grau de curtose platicúrtica $(K=2,51)$, caracterizada pelo valor médio de 140,18 US $\$ \mathrm{~h}^{-1}$ e desvio padrão de 30,15 US $\$ h^{-1}$.

Ao expressar as classificações das associações dos $E C$, ficou evidente que $o C_{\text {pec }}$ possui uma correlação não linear forte positiva com o $\operatorname{CHP}\left(\rho_{s}=0,92\right)$, isto é, quanto maior o dispêndio monetário com peças, maior será o CHP, além disto, o este $C_{\text {pec }}$ exerceu uma influência de $85,39 \%$ sobre a variação do valor médio do CHP. Ainda sob este prisma, o $C_{\text {rem }}$ também apresenta uma correlação não linear moderada positiva $\operatorname{com} \circ \operatorname{CHP}\left(\rho_{s}=0,71\right)$, provocando um impacto de $11,49 \%$ sobre o valor médio do CHP.

Apesar do $C_{\text {lub }}$ apresentar um grau de correlação que pode ser considerado fraco, ressalta-se que este é negativo (Figura 3), quer dizer, um aumento dos gastos monetários com lubrificantes poderia diminuir o custo por hora programada, o que indica que a lubrificação preventiva e preditiva da máquina autopropelida permitiria uma redução dos gastos com reposição de peças e gastos com reparos e manutenções.

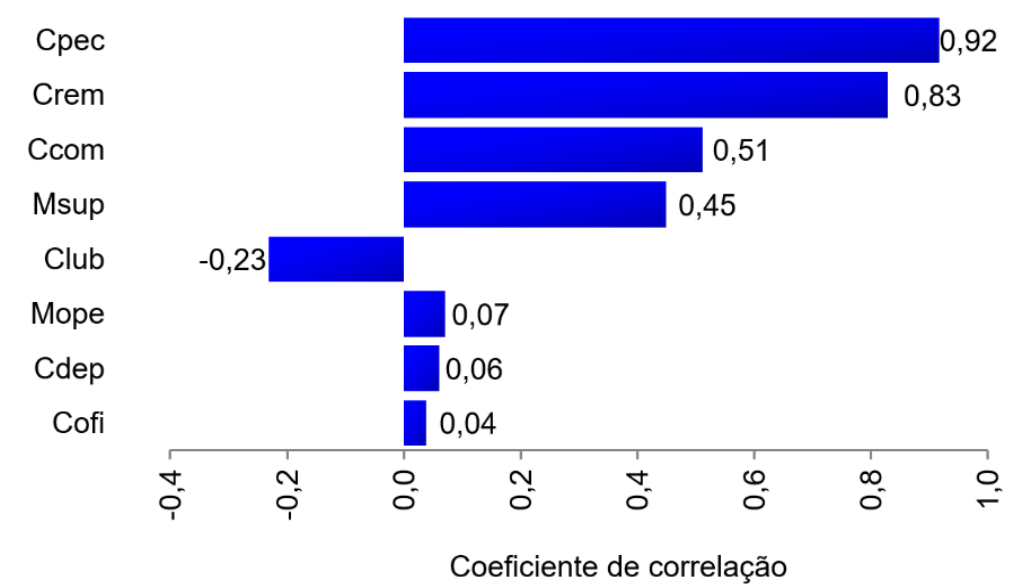

Figura 3. Coeficiente de correlação de postos de Spearman dos elementos de custo da máquina autopropelida com cabeçote de corte com disco feller-buncher durante a derrubada de Eucalyptus sp. na Área 1.

Em relação ao CHP da derrubada de Eucalyptus sp. na Área 2, comprovou-se que os valores não seguem uma distribuição Normal $(K S=0,0161)$. Além disto, a distribuição de probabilidade que melhor se ajustou aos dados (BIC=964.827,49) foi a Beta(BIC=964.827,49), com assimetria fraca e positiva $(A=0,49)$ e com cauda mais longa à direita, e grau de curtose platicúrtica $(K=2,57)$. O valor médio foi152,76 US\$ $\mathrm{h}^{-1}$ com desvio padrão de31,92 US\$ $\mathrm{h}^{-1}$.

É possível observar na Figura 4 que houve uma correlação positiva forte $\left(\rho_{s}=0,92\right)$ do $C_{\text {pec }}$ com o CHP, de outra forma, este EC contribuiu $85,17 \%$ para a variância do valor médio CHP. Outro EC que que também contribuiu para variância $(11,62 \%)$ do valor médio do $C H P$ foi o $C_{\text {rem }}$ que apresentou uma correlação positiva, contudo, considerada moderada $\left(\rho_{s}=0,71\right)$. 


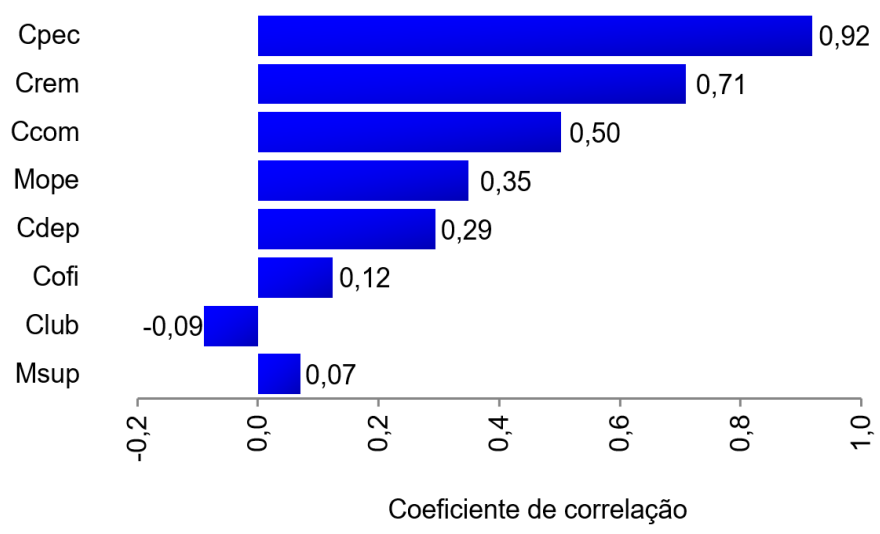

Figura 4. Coeficiente de correlação de postos de Spearman dos elementos de custo da máquina autopropelida com cabeçote de corte com disco feller-buncher durante a derrubada de Eucalyptus sp. na Área 2.

A relação não linear $C_{\text {lub }}$ com o CHP também teve um comportamento análogo em florestas plantadas com Eucalyptus sp. na Área 1, ou seja, a tendência dos inputs é mover-se na direção oposta, pois a correlação não linear foi negativa (Figura 4), ainda que, caracterizada como fraca.

\section{Análise estocástica do custo de produção da máquina autopropelida}

O estudo pormenorizado do COM da derrubada de Eucalyptus sp. na Área 1, aferiu que o pressuposto de normalidade foi rejeitado (KS=0,0018). Assim, o melhor ajuste para os dados observados (Figura 5) foi a distribuição Log-normal $(B I C=84.171,98)$ com assimetria fraca e positiva $(A=1,26)$ e com cauda mais longa à direita e grau de curtose platicúrtica $(K=6,86)$. Ainda, o valor médio do CPM foi 1,32 US $\$ \mathrm{~m}^{-3} \mathrm{e}$ desvio padrão 0,41 US $\$ \mathrm{~m}^{-3} \mathrm{e}$, notou-se, que a variância do valor médio do CPM (46,86\%) foi explicada pela PHM.

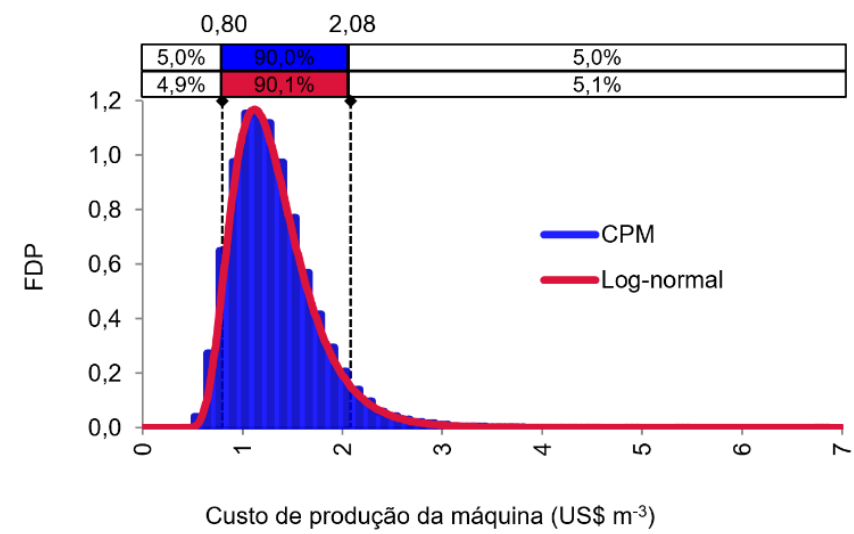

Figura 5. Função densidade de probabilidade (FDP) do custo de produção da máquina autopropelida com cabeçote de corte com disco feller-buncher durante a derrubada de Eucalyptus sp. na Área 1.

Com referência ao COM da derrubada de Eucalyptus sp. na Área 2, constatou-se que a hipótese de normalidade dos dados foi rejeitada $(K S=0,0109)$. Pautado no BIC, observou-se que o melhor ajuste da distribuição de probabilidade foi a Log-normal (BIC=219.308,06), a qual permitiu a modelagem assimétrica dos dados (Figura 6), pois resultou em uma assimetria fraca e positiva com cauda mais longa a direita $(A=1,81)$ e com grau de curtose platicúrtica $(K=8,44)$, sendo o valor médio do CPM de 1,39 US $\$ \mathrm{~m}^{-3}$ comdesvio padrão de $0,63 \cup S \$ \mathrm{~m}^{-3} \mathrm{e}$, em termos de variância do valor médio $(45,26 \%)$ explicada pela PHM. 


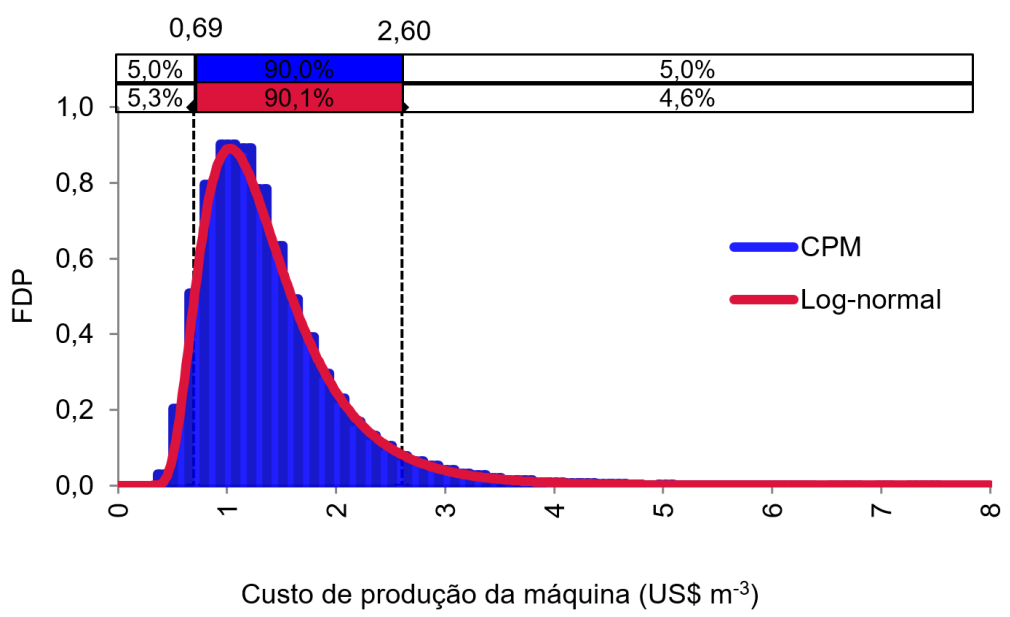

Figura 6. Função densidade de probabilidade (FDP) do custo de produção da máquina autopropelida com cabeçote de corte com disco feller-buncher durante a derrubada de Eucalyptus sp. na Área 2.

Ao colacionar o valor médio do CPM obtido para ambas as áreas experimentais $\left(1,35\right.$ US $\left.\$ \mathrm{~m}^{-3}\right)$ de forma empírica com os custos de produção de 0,72 US $\$ \mathrm{~m}^{-3}, 0,71 \cup S \$ \mathrm{~m}^{-3}$, 0,75 US $\$ \mathrm{~m}^{-3}$ e 0,58 US $\$ \mathrm{~m}^{-3}$, concomitantemente, determinado por Simões et al. (2014) Pereira et al. (2015), Santos et al. (2018b) e Simões et al. (2018) a partir de estimativas dos valores técnico-econômicos para a obtenção do custo por hora programada da máquina autopropelida realizadas por estes autores, pode ser assumida diferenças dos valores monetários.

No entanto, estas diferenças não representam equívocos, visto que as distintas possibilidades de cálculo do custo por hora programada da máquina autopropelida não são totalmente precisas e podem apresentar disparidades. Por esse motivo, o método de custeio a ser empregado deve estar em consonância aos centros de custos das empresas de base florestal, visando a determinação dos custos de produção próximos às condições realistas.

\section{CONCLUSÕES}

Os valores monetários demandados para a substituição de peças da máquina autopropelida com cabeçote de corte com disco feller-buncher possui o maior grau de associação com o custo por hora programada, descrevendo uma forte relação positiva.

Há uma relação não linear forte negativa entre os valores monetários necessários para a aquisição de óleos lubrificantes e graxas e entre o custo por hora programada, evidenciando que quanto maior o investimento em lubrificação menor será o custo por hora programada da máquina autopropelida.

O valor médio do custo de produção para a derrubada de Eucalyptussp. é explicado por $46 \%$ em termos de variância da produtividade por hora efetivada máquina autopropelida com cabeçote de corte com disco feller-buncher.

\section{REFERÊNCIAS BIBLIOGRÁFICAS}

Acosta, F. C., Laroca, J. V. S., Santos, O. I. V., \& Silva, J. P. P. (2017). Productividad y costo del corte de árboles com feller buncher en plantaciones de Eucalyptus. Nativa, 5(3), 218-223.

Alam, M., Acuna, M., \& Brown, M. (2013). Self-levelling feller-buncher productivity based on Lidarderived slope. Croatian Journal of Forest Engineering: Journal for Theory and Application of Forestry Engineering, Zagreb, 34(2), 273-281.

Banco Central do Brasil (2018). Conversão de moedas. Retrieved in 2018, May 01, from http://www4.bcb.gov.br/pec/conversao/conversao.asp

Bilici, E., Akay, A. E., \& Abbas, D. (2018). Assessing the effects of site factors on the productivity of a feller buncher: a time and motion analysis. Journal of Forestry Research, 30, 1471-1478. 
Buhara, O., \& Pehlivan, S. (2019). Monte Carlo simulation of reasons for early failure of implants: effects of two risk factors. British Journal of Oral and Maxillofacial Surgery, 57(1),12-20.

Daily, J., \& Peterson, J. (2017). Predictive maintenance: How big data analysis can improve maintenance. In K. Richter \& J. Walther. Supply Chain Integration Challenges in Commercial Aerospace (p. 267-278). Cham: Springer International Publishing. http://dx.doi.org/10.1007/978-3-319-46155-7_18.

Fernandes, H. C., Lopes, S. E., Teixeira, M. M., Minette, L. J., Rinaldi, P. C. N., \& Bernardes, A. M. (2009). Avaliação das características técnica e econômica de um sistema de colheita florestal de árvores inteiras. Scientia Forestalis, 37(83), 225-232.

Fernandes, H. C., Brito, A. B., Minetti, L. J., Santos, N. T., \& Rinaldi, P. C. N. (2010). Avaliação ergonômica da cabine de um trator florestal. Revista Ceres, 57(3), 307-314.

Fiedler, N. C., Rocha, E. B., \& Lopes, E. S. (2008). Análise da produtividade de um sistema de colheita de árvores inteiras no norte do estado de Goiás. Floresta, 38(4), 577-586.

Ghorabaee, M. K., Amiri, M., Zavadskas, E. K., Turskis, Z., \& Antucheviciene, J. (2017). A new hybrid simulation-based assignment approach for evaluating airlines with multiple service quality criteria. Journal of Air Transport Management, 63, 45-60. http://dx.doi.org/10.1016/j.jairtraman.2017.05.008.

Glickman, T. S., \& Xu, F. (2008). The distribution of the product of two triangular random variables. Statistics \& Probability Letters, 78(16), 2821-2826.

Instituto Geográfico e Cartográfico do Estado de São Paulo - ICG (2018). Produtos. Retrieved in 2018, June 12, from http://www.igc.sp.gov.br/servicos/index.html

Jing, L., Chen, B., Zhang, B., Li, P., \& Zheng, J. (2013). Monte Carlo simulation-aided analytic hierarchy process approach: Case study of assessing preferred non-point-source pollution control best management practices. Journal of Environmental Engineering (New York), 139(5), 618-626.

Kabungo, A. M., \& Jenkins, G. P. (2016). Contract farming risks: A quantitative assessment. South African Journal of Economic and Management Sciences, Pretoria, 19(1), 35-52.

Kamiya, H., Cho, B. H., Messonnier, M. L., Clark, T. A., \& Liang, J. L. (2016). Impact and cost-effectiveness of a second tetanus toxoid, reduced diphtheria toxoid, and acellular pertussis (Tdap) vaccine dose to prevent pertussis in the United States. Vaccine. Kidlinton, 34(15), 1832-1838. PMid:26899377. http://dx.doi.org/10.1016/j.vaccine.2016.02.027.

Mauya, E. W., Migunga, G. A., Kweka, A. E., \& Silayo, D. A. (2011). Productivity and cost analysis of feller buncher at Sao Hill Forest Plantations, Tanzania. Tanzania Journal of Forestry and Nature Conservation, Morogoro, 81(1), 20-29.

Miguel, E. P., Leal, F. A., Ono, H. A. \& Leal, U. A. S. (2014). Modelagem na predição do volume individual em plantio de Eucalyptus urograndis. Revista Brasileira de Biometria, 32(4), 584-598.

Miyajima, R. H., Tonin, R. P., Passos, J. R. S., \& Fenner, P. T. (2016). A Influência da declividade do terreno e do tempo de experiência dos operadores no rendimento do feller buncher. Scientia Forestalis, 44(110), 443-451.

Miyajima, R. H., Tonin, R. P., Fenner, P. T., \& Simões, D. (2017). Análise quantitativa do risco técnicoeconômico de um trator florestal skidder. BIOFIX Scientific Journal. 2(Especial I MECFOR 2017), 6-11.

Moreira, F. M. T., Souza, A. P., Machado, C. C., Minetti, L. J., \& Silva, K. R. (2004). Avaliação operacional e econômica do "feller-buncher" em dois subsistemas de colheita de florestas de eucalipto. Revista Árvore, 28(2), 199-205.

Nguyen, H. D., \& McLachlan, G. J. (2016a). Maximum likelihood estimation of triangular and polygonal distributions. Computational Statistics \& Data Analysis, 102, 23-36.

Nguyen, H. D., \& McLachlan, G. J. (2016b). Progress on a conjecture regarding the triangular distribution. Communications in Statistics-Theory and Methods, 46(22), 11261-11271.

Palisade Corporation (2018). Palisade Corporation @Risk for Excel. v. 7.6.0. Ithaca, NY: Palisade Corporation.

Parra, G. G., Arenas, A. J., \& Cogollo, M. (2015). Analytical-numerical solution of a parabolic diffusion equation under uncertainty conditions using DTM with Monte Carlo simulations. Ingeniería y Ciencia, $11(22), 49-72$.

Pereira, A. L. N., Silva Lopes, E., \& Dias, A. N. (2015). Análise técnica e de custo do feller buncher e skidder na colheita de madeira em diferentes produtividades do povoamento. Ciência Florestal 25(4), 981-989. http://dx.doi.org/10.5902/1980509820659.

Porras, A. F. (2018). Cálculo de la actividad de una muestra de uranio irradiada por neutrones térmicos para el análisis inicial en protección radiologica usando simulación Monte Carlo. Momento, (56), 7686. 
Rachev, S. T., Höchstötter, M., Fabozzi, F. J., \& Focardi, S. M. (2010). Probability and statistics for finance (654 p.). Hoboken: John Wiley \& Sons, Inc. http://dx.doi.org/10.1002/9781118267912.

Santos, D. W. F. N., Fernandes, H. C., Valente, D. S. M., \& Leite, E. S. (2018b). Análise técnica e econômica de dois subsistemas de colheita de madeira de toras curtas. Revista Brasileira de Ciências Agrárias, 13(2), 1-6. http://dx.doi.org/10.5039/agraria.v13i2a5516.

Sant'Anna, C. M. (2014). Corte. In Machado, C.C. (Org.). Colheita Florestal (3. ed., pp. 74-105). Viçosa, MG: Editora UFV.

Santos, H. G., Jacomine, P. K. T., Anjos, L. H. C., Oliveira, V. A., Lumbreras, J. F., Coelho, M. R., Almeida, J. A., Araujo Filho, J. C., Oliveira, J. B., \& Cunha, T. J. F. (2018a). Sistema brasileiro de classificação de solos (5. ed., 356 p.). Brasília, DF: EMBRAPA.

Silva, J. R., Fenner, P. T., \& Cataneo, A. (2007). Avaliação do desempenho operacional de trator florestal Feller-buncher. Revista de Ciências Agroveterinária, 6(1), 29-34.

Simões, D., Fenner, P. T., \& Esperancini, M. S. T. (2014). Produtividade e custos do feller-buncher e processador florestal em povoamento de eucalipto de primeiro corte. Ciência Florestal, 24(3), 621630.

Simões, D., lamonti, I. C., \& Fenner, P. T. Avaliação técnica e econômica do corte de eucalipto com fellerbuncher em diferentes condições operacionais. Ciência Florestal, 20(4), 649-656, 2010.

Simões, D., Miyajima, R. H., Tonin, R. P., Fenner, P. T., \& Batistela, G. C. (2018). Incorporation of uncertainty in technical and economic analysis of a feller-buncher. Floresta, 48(3), 403-412.

Sullivan, M. (2017). Statistics: informed decisions using data plus MyStatLab with Pearson eText. (5th ed., 960 p.). Boston: Pearson Education, Inc.

Triola, M. F. (2015). Essentials of statistics. (5th ed., 669 p.). Boston: Pearson Education, Inc.

Contribuição dos autores: GP: investigação, escrita - primeira redação; PTF: supervisão; GCB: validação, análise formal; DS: conceituação, metodologia, escrita - revisão e edição. 